\title{
A rigorous proof of non-existence of edge state in the semi-infinite armchair edged graphene
}

\author{
Yuanyuan Zhao, Wei Li and Ruibao Tao \\ State Key Laboratory of Surface Physics and Department of Physics, \\ Fudan University, Shanghai, 200433, People's Republic of China
}

November 9, 2018

\begin{abstract}
With the help of transfer matrix method, the conditions for the existence of the edge states in the semi-infinite armchair edged graphene is given. We discuss zero-energy and non-zero-energy edge states separately, and show the nonexistence of the edge states in the model analytically and rigorously.
\end{abstract}

PACS: 73.22.Pr, 73.20.At, 71.15.-m

\section{Introduction}

One of the interesting phenomena in solid state physics is the existence of edge states on the boundary, the properties of which are distinct from those of the bulk states, and they can play an important role in transport, there are examples showing that system is insulated in the bulk, while conduction can be allowed by edge states on the boundary. The most prominent ones are the quantum Hall effect (QHE) 1, 2, 3, 4] and the quantum spin Hall effect (QSHE) [5, 6, 7], where the quantization of a Hall conductance is tightly associated with the edge states 2 , 3, 4, 4, 6, 7, 8, 9, 10.

The realization of graphene[11, 12, 13] in laboratories has triggered great research interests in recent years. From the topological viewpoint, edge states can be induced in the system with different edge geometries [14, 15]; in experiments, with the help of scanning tunneling microscopy and scanning tunneling spectroscopy, the presence of structure-dependent edge states of graphite can be observed [16, 17]. There are some papers [18, 19] showing the similarity between the graphene model and the $d$-wave superconductor [20] with edges, where the existence of edge states depends on its edge shapes. For comparison, on a $\{110\}$ surface of a $d_{x^{2}-y^{2}}$-wave superconductor, the origin of the Andreev bound states is the $\pi$-phase shift due to the unconventional pairing symmetry, and graphene can be seem as an odd-parity 
superconductor [19], at the zigzag/bearded edges, different signs of pairing potential lead to a $\pi$-phase shift and zero-energy bound states, while at the armchair edge, it will not show the localized edge states. Hence the study on the zero mode of the edge state will have the fundamental meaning that can supply some information about the phase structure.

The purpose of this paper is to study the edge states of the semi-infinite armchair edged graphene (AEG) analytically with the help of transfer matrix method [21, 22, 23, 24, 25]. There are many papers showing the edge states in graphene models in different ways, while most of them focus on the zero mode of the zigzag graphene nanoribbon. And here we pay our attention on the semi-infinite armchair edged graphene, and study the zero-energy and non-zero-energy edge states. According to the discussions of the properties of the transfer matrix, we show an analytically proof of the non-existence of the edge states in the semi-infinite AEG. We have to mention that we just consider about nearest-neighbor-interaction in our whole discussions.

\section{EDGE STATES OF SEMI-INFINITE AEG}

The geometrical structure of graphene is shown in Fig 1. It is infinite in $y$ direction with periodic constant $\sqrt{3} a$, here $a$ is the lattice constant between two n.n. A(B) atoms. We arrange all vertical chains in the order from the left to the right as $\{1,2,3, \cdots\}$. Each vertical chain is an $1 \mathrm{D}$ atomic chain, in which the periodic cell contains two kinds of atoms $A$ and $B$. The position of the atoms are labeled by two indices $(n, j)$, where $n$ labels the order number of vertical chains from 1 to $\infty$, with $n=1$ representing the left edge.

Due to its infinity in $y$ direction, $k_{y}$ is a good quantum number, so that we can take the Fourier transformation on wave functions $\Psi_{A(B)}^{(n, j)}\left(k_{y}\right)$ for $A(B)$ atoms: $\Psi_{A(B)}^{(n, j)}\left(k_{y}\right)=\exp \left(i k_{y} y_{j, A(B)}\right) \Phi_{n, A(B)}\left(k_{y}\right)$. The Hamiltonian now in $\left\{n, k_{y}\right\}$ representation can be expressed by a set of Fermion operators $\left\{\Phi_{n, A(B)}\left(k_{y}\right)\right\}, n=1,2, \ldots, \infty$ :

$$
\begin{aligned}
H & =t \sum_{k_{y}, n \geqslant 1} e^{i k_{y} a / \sqrt{3}} \Phi_{n, A}^{\dagger}\left(k_{y}\right) \Phi_{n, B}\left(k_{y}\right) \\
& +t \sum_{k_{y}, n \geqslant 2} e^{-i k_{y} a / 2 \sqrt{3}} \Phi_{n, A}^{\dagger}\left(k_{y}\right) \Phi_{n-1, B}\left(k_{y}\right) \\
& +t \sum_{k_{y}, n \geqslant 1} e^{-i k_{y} a / 2 \sqrt{3}} \Phi_{n, A}^{\dagger}\left(k_{y}\right) \Phi_{n+1, B}\left(k_{y}\right)+\text { h.c. }
\end{aligned}
$$

From the dynamical equations of $\left\{\Phi_{n, A(B)}\left(k_{y}\right)\right\}: E \Phi_{n, A(B)}\left(k_{y}\right)=\left[\Phi_{n, A(B)}\left(k_{y}\right), H\right]$, 


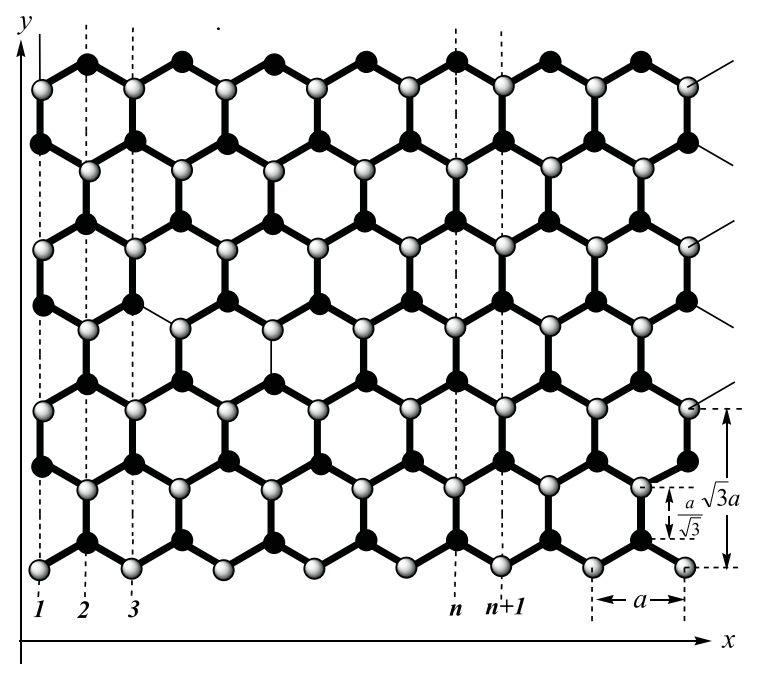

Figure 1: Schematic illustration of the lattice structure of semi-infinite armchair edged graphene. The black circles represent the type $B$ atom, while empty circle represents type $A$ atom; It is infinite in $y$ direction and the edge locates at the left perpendicular chain labeled by number 1 .

we get following recursive equations for each fixed wave vector $k_{y}$ :

$$
\left\{\begin{aligned}
E \Phi_{n, A} & =t e^{i k_{y} a / \sqrt{3}} \Phi_{n, B}+t e^{-i k_{y} a / 2 \sqrt{3}} \Phi_{n-1, B} \\
& +t e^{-i k_{y} a / 2 \sqrt{3}} \Phi_{n+1, B}, \quad n=2,3, \cdots \\
E \Phi_{n, B} & =t e^{-i k_{y} a / \sqrt{3}} \Phi_{n, A}+t e^{i k_{y} a / 2 \sqrt{3}} \Phi_{n-1, A} \\
& +t e^{i k_{y} a / 2 \sqrt{3}} \Phi_{n+1, A}, \quad n=2,3, \cdots
\end{aligned}\right.
$$

where we use $\Phi_{n, A(B)}$ instead of $\Phi_{n, A(B)}\left(k_{y}\right)$. The equations of the wave functions for the boundary sites $(n=1)$ are

$$
\left\{\begin{array}{l}
E \Phi_{1, A}=t e^{i k_{y} a / \sqrt{3}} \Phi_{1, B}+t e^{-i k_{y} a / 2 \sqrt{3}} \Phi_{2, B}, \\
E \Phi_{1, B}=t e^{-i k_{y} a / \sqrt{3}} \Phi_{1, A}+t e^{i k_{y} a / 2 \sqrt{3}} \Phi_{2, A} .
\end{array}\right.
$$

We can get the bulk energy for the extended states, according to Eq. (2), take $k_{x}$ as good quantum number, the bulk energy satisfies:

$$
E^{2}=1+4 \cos ^{2}\left(k_{x} a / 2\right)+4 \cos \left(\sqrt{3} k_{y} a / 2\right) \cos \left(k_{x} a / 2\right)
$$

which is the energy dispersion relation of the $2 \mathrm{D}$ graphene system. In the following, we will focus on the edge states, and discuss the zero-energy ( $E=$ $0)$ and non-zero-energy $(E \neq 0)$ cases, separately. To examine whether the edge states can exist in the semi-infinite graphene model or not. 
(1) $E=0$ : the zero-energy spectrum. The recursions Eq. (2) are reduced into two decoupled equations for sublattice $A$ and $B$ :

$$
\left\{\begin{aligned}
\Phi_{n+1, \alpha} & =-e^{-i \eta_{\alpha} \sqrt{3} k_{y} a / 2} \Phi_{n, \alpha}-\Phi_{n-1, \alpha} \\
\Phi_{2, \alpha} & =-e^{-i \eta_{\alpha} \sqrt{3} k_{y} a / 2} \Phi_{1, \alpha}, \\
& \alpha=A, B, \eta_{A}=1, \eta_{B}=-1, \quad n=2,3, \cdots .
\end{aligned}\right.
$$

Above equations can be rewritten as follows transfer matrix equations:

$$
\left(\begin{array}{c}
\Phi_{n+2, \alpha} \\
\Phi_{n+1, \alpha}
\end{array}\right)=T_{\alpha}^{n}\left(\begin{array}{c}
b_{\alpha} \\
1
\end{array}\right) \Phi_{1, \alpha}, n=1,2,3, \cdots .
$$

where

$$
b_{\alpha}=-e^{-i \eta_{\alpha} \sqrt{3} k_{y} a / 2}
$$

$$
T_{\alpha}=\left(\begin{array}{cc}
-e^{-i \eta_{\alpha} \sqrt{3} k_{y} a / 2} & -1 \\
1 & 0
\end{array}\right)
$$

$\operatorname{det} T_{\alpha}=1$. It is easy to get two eigenvalues of $T_{\alpha}(\alpha=A, B): \lambda_{ \pm}^{(\alpha)}=$ $\frac{1}{2} \Lambda_{\alpha} \pm \frac{1}{2} i \sqrt{4-\Lambda_{\alpha}^{2}}$ where $\Lambda_{\alpha}=e^{-i \eta_{\alpha} k_{y} a \sqrt{3} / 2} .\left|\Lambda_{\alpha}\right|=1$ and two eigenvalues $\lambda_{ \pm}^{(\alpha)}$ must be complex and $\lambda_{ \pm}^{\alpha}=\left(\lambda_{\mp}^{\alpha}\right)^{*}$. It results $\left|\lambda_{ \pm}^{\alpha}\right|=1$, due to $\lambda_{+}^{\alpha} \cdot \lambda_{-}^{\alpha}=1$. Thus, we can conclude that the states are always extended in the semiinfinite AEG, no zero-energy edge state can exist.

(2) $E \neq 0$ : according to Eq. (2), the equations of two sublattices are coupled with each other. We can also write down a transfer matrix for $A$ or $B$ type sublattices, and it becomes $4 \times 4$. For more concise description, we define a $4 \times 1$ vector $\Psi_{\alpha}^{(n)}$, its transpose $\left(\Psi_{\alpha}^{(n)}\right)^{t}$ is a $1 \times 4$ vector as $\left(\begin{array}{cccc}\Phi_{n, \alpha} & \Phi_{n-1, \alpha} & \Phi_{n-2, \alpha} & \Phi_{n-3, \alpha}\end{array}\right)$. Then, the Eq. (2) can be expressed as follows:

$$
\left\{\begin{array}{l}
\Psi_{\alpha}^{(n+2)}=T_{0} \Psi_{\alpha}^{(n+1)}, n \geqslant 3 \\
T_{0}=\left(\begin{array}{cccc}
G & \lambda & G & -1 \\
1 & 0 & 0 & 0 \\
0 & 1 & 0 & 0 \\
0 & 0 & 1 & 0
\end{array}\right), \quad \operatorname{det} T_{0}=1,
\end{array}\right.
$$

where $G=-2 \theta, \theta=\cos \left(\sqrt{3} k_{y} a / 2\right), \lambda=\left(E^{2}-3 t^{2}\right) / t^{2}=E^{2}-3($ set $t=1)$. We further introduce one fictitious lattice line at left of edge chain and set them all to be zero: $\Phi_{\alpha}^{(0)}=0$. It will not change anything even introducing the hopping coupling with $1 s t$ line. Now Eq. (2) and Eq. (3) can be written as follows:

$$
\begin{aligned}
& \Psi_{\alpha}^{(n)}=T_{0}^{n-3} \Psi_{\alpha}^{(3)}, \quad n \geqslant 4 . \\
& \Psi_{\alpha}^{(3)}=G \Psi_{\alpha}^{(2)}+(\lambda+1) \Psi_{\alpha}^{(1)} .
\end{aligned}
$$


the eigenvalues of transfer matrix $T_{0}$ can be written in the form of $\left\{\lambda_{i}\right\}$ :

$$
\begin{aligned}
& \lambda_{1}=\frac{1}{2}\left(-a_{1}-\sqrt{a_{1}^{2}-4}\right), \lambda_{2}=\frac{1}{2}\left(-a_{1}+\sqrt{a_{1}^{2}-4}\right), \\
& \lambda_{3}=\frac{1}{2}\left(-a_{2}-\sqrt{a_{2}^{2}-4}\right), \lambda_{4}=\frac{1}{2}\left(-a_{2}+\sqrt{a_{2}^{2}-4}\right),
\end{aligned}
$$

where

$$
a_{1}=\theta+\sqrt{m}, a_{2}=\theta-\sqrt{m}, m=\lambda+2+\theta^{2} .
$$

Now, define a transformation $U$, where $U^{-1}$ exists, to rewrite the transfer matrix and its relation Eq. (9) with $D=U^{-1} T_{0} U=\operatorname{diag}\left\{\lambda_{1}, \lambda_{2}, \lambda_{3}, \lambda_{4}\right\}$. In order to get the physically meaningful states, we have to ensure that all eigenvalues $\left\{\left|\lambda_{i}\right|\right\}$ must be finite for any finite energy $E$.

When $m \neq 0, a_{1}^{2} \neq 4$, and $a_{2}^{2} \neq 4$, we can write down $U$ as follows

$$
U=\left(\begin{array}{cccc}
\lambda_{1}^{3} & \lambda_{2}^{3} & \lambda_{3}^{3} & \lambda_{4}^{3} \\
\lambda_{1}^{2} & \lambda_{2}^{2} & \lambda_{3}^{2} & \lambda_{4}^{2} \\
\lambda_{1} & \lambda_{2} & \lambda_{3} & \lambda_{4} \\
1 & 1 & 1 & 1
\end{array}\right)
$$

with $\operatorname{det} U \neq 0$. Its inverse matrix $U^{-1}$ can be found and its elements of $U_{i j}^{-1}$ are described as follows

$$
\left\{\begin{array}{l}
U_{i j}^{-1}=C_{i j} / u_{i}\left(a_{1}-a_{2}\right), \\
u_{1}=a_{1}^{2}-4+a_{1} \sqrt{a_{1}^{2}-4}=-2 \lambda_{1} \sqrt{a_{1}^{2}-4} \\
u_{2}=4-a_{1}^{2}+a_{1} \sqrt{a_{1}^{2}-4}=-2 \lambda_{2} \sqrt{a_{1}^{2}-4} \\
u_{3}=a_{2}^{2}-4+a_{2} \sqrt{a_{2}^{2}-4}=-2 \lambda_{3} \sqrt{a_{2}^{2}-4} \\
u_{4}=a_{2}^{2}-4-a_{2} \sqrt{a_{2}^{2}-4}=2 \lambda_{4} \sqrt{a_{2}^{2}-4}
\end{array}\right.
$$

where

$$
\left\{\begin{array}{l}
C_{41}=C_{31}=C_{21}=-C_{11}=2, \\
C_{12}=\sqrt{a_{1}^{2}-4}-a_{1}-2 a_{2}, \\
C_{22}=\sqrt{a_{1}^{2}-4}+a_{1}+2 a_{2} \\
C_{13}=-2-a_{1} a_{2}+a_{2} \sqrt{a_{1}^{2}-4} \\
C_{23}=2+a_{1} a_{2}+a_{2} \sqrt{a_{1}^{2}-4} \\
C_{33}=2+a_{1} a_{2}-a_{1} \sqrt{a_{2}^{2}-4} \\
C_{43}=2+a_{1} a_{2}+a_{1} \sqrt{a_{2}^{2}-4} \\
C_{32}=2 a_{1}+a_{2}-\sqrt{a_{2}^{2}-4} \\
C_{42}=2 a_{1}+a_{2}+\sqrt{a_{2}^{2}-4} \\
C_{14}=-a_{1}+\sqrt{a_{1}^{2}-4}, \quad C_{24}=a_{1}+\sqrt{a_{1}^{2}-4} \\
C_{34}=a_{2}-\sqrt{a_{2}^{2}-4}, \quad C_{44}=a_{2}+\sqrt{a_{2}^{2}-4}
\end{array}\right.
$$


The relation $U^{-1} T_{0} U=D$ was carefully confirmed by $U$ and $U^{-1}$. After tedious but straightforward algebraic calculations, we obtain

$$
\Psi_{\alpha}^{(n+3)}=U D^{n} U^{-1} \Psi_{\alpha}^{(3)}=U\left(\begin{array}{l}
W_{1} \lambda_{1}^{n} \\
W_{2} \lambda_{2}^{n} \\
W_{3} \lambda_{3}^{n} \\
W_{4} \lambda_{4}^{n}
\end{array}\right), n \geqslant 1 .
$$

Where

$$
\begin{aligned}
W_{i}= & \left(U_{i 2}^{-1}+G U_{i 1}^{-1}\right) \Phi_{2, \alpha}+\left(U_{i 3}^{-1}+(\lambda+1) U_{i 1}^{-1}\right) \Phi_{1, \alpha}, \\
& i=1,2,3,4 .
\end{aligned}
$$

Since all elements of matrix $U_{i j}$ are non-zero and finite, thus the criteria whether the state $\Phi_{n, \alpha}$ is extended or edge state can be determined by those $\left\{W_{i} \lambda_{i}^{n}\right\}$ regardless the front matrix $U$ in Eq. (17).

From the definition of $\left\{\lambda_{i}\right\}$, we can confirm $\lambda_{1} \cdot \lambda_{2}=1$ and $\lambda_{3} \cdot \lambda_{4}=1$. thus $\left|\lambda_{2}\right|=\left|\lambda_{1}\right|^{-1}$ and $\left|\lambda_{3}\right|=\left|\lambda_{4}\right|^{-1}$. Define $\left\{\lambda_{1}, \lambda_{2}\right\}\left(\left\{\lambda_{3}, \lambda_{4}\right\}\right)$ as a pair partner. $\lambda_{2(1)}$ is the partner of $\lambda_{1(2)}$, while $\lambda_{4(3)}$ the partner of $\lambda_{3(4)}$. Then we use $\lambda_{\bar{i}}$ to denote the partner of $\lambda_{i}$. For example, $\lambda_{2}=\lambda_{\overline{1}}$ and $\lambda_{1}=\lambda_{\overline{2}}$. It is similar to define the partner of $W_{i}$ [see Eq. (17) and Eq. (18)]: the partner of $W_{1(2)}$ is $W_{2(1)}=W_{\overline{1}(\overline{2})}$, and $W_{3(4)}$ the $W_{4(3)}=W_{\overline{3}(\overline{4})}$. If $\left|\lambda_{i}\right|=1$, we do have $\left|\lambda_{\bar{i}}\right|=1$, which corresponds to the extended states. As we know, the edge states correspond to some $\left|\lambda_{i}\right|<1$, due to the properties of $\lambda_{i}$ and its partner $\lambda_{\bar{i}}$, in order to get the physically meaningful non-zero-energy edge states, the amplitude of which must be finite at $n \rightarrow \infty$ in Eq. (17), so that we can get the necessary condition for the edge states $W_{i}=0$ and $W_{\bar{i}} \neq 0$ when $\left|\lambda_{i}\right|>1$ and $\left|\lambda_{\bar{i}}\right|<1$.

Without loss of generality, at first we assume $\left|\lambda_{1}\right|>1$ at some $\left\{E, k_{y}\right\}$. In the case, we have $\left|\lambda_{2}\right|<1$. The necessary condition for the existence of edge state is $W_{1}=0$ and $W_{2}=\eta \neq 0$. From Eq. (18), the necessary condition turns to

$$
\left\{\begin{array}{l}
A_{11} \Phi_{2, \alpha}+A_{12} \Phi_{1, \alpha}=0 \\
A_{21} \Phi_{2, \alpha}+A_{22} \Phi_{1, \alpha}=\eta(\neq 0)
\end{array}\right.
$$

Where

$$
\begin{cases}A_{11}=U_{12}^{-1}+G U_{11}^{-1}, & A_{12}=U_{13}^{-1}+(\lambda+1) U_{11}^{-1}, \\ A_{21}=U_{22}^{-1}+G U_{21}^{-1}, & A_{22}=U_{23}^{-1}+(\lambda+1) U_{21}^{-1},\end{cases}
$$


In terms of relations (15), (16) and (13), we get

$$
\begin{aligned}
& A_{11}=\frac{C_{12}+G C_{11}}{u_{1}\left(a_{1}-a_{2}\right)}=\frac{1}{2 \sqrt{a_{1}^{2}-4} \sqrt{m}}, \\
& A_{12}=\frac{C_{13}+(\lambda+1) C_{11}}{u_{1}\left(a_{1}-a_{2}\right)}=\frac{a_{2}}{2 \sqrt{a_{1}^{2}-4} \sqrt{m}}, \\
& A_{21}=\frac{C_{22}+G C_{21}}{u_{2}\left(a_{1}-a_{2}\right)}=\frac{-1}{2 \sqrt{a_{1}^{2}-4} \sqrt{m}} \\
& A_{22}=\frac{C_{23}+(\lambda+1) C_{21}}{u_{2}\left(a_{1}-a_{2}\right)}=\frac{-a_{2}}{2 \sqrt{a_{1}^{2}-4} \sqrt{m}} .
\end{aligned}
$$

where the relations Eq. (12), Eq. (13), Eq. (15), and Eq. (16) have been used. According to $m \neq 0$ and $a_{1}^{2} \neq 4$ and $a_{2}^{2} \neq 4, A_{11}$ and $A_{21}$ both are finite and not zero. If $a_{2}=0$, we have $A_{12}=A_{22}=0$. The 1 st Eq. (19) must lead to $\Phi_{2, \alpha}=0$ because of $A_{11} \neq 0$, meanwhile the 2 nd Eq. (19) showing $A_{22} \Phi_{1, \alpha}=\eta \neq 0$ could not be satisfied. Thus $a_{2}=0$ must result $W_{2}=0$. Assume all elements $\left\{A_{i j}\right\}$ are not zero $\left(a_{2} \neq 0\right)$ in our following discussion. To ensure that both equations in Eq. (19) should be satisfied with $\eta \neq 0$, we can write down a non-homogeneous linear equations with vector $\left(\Phi_{2, \alpha}, \Phi_{1, \alpha}\right)^{t}$, therefore Eq. (19) can be written as

$$
A\left(\begin{array}{l}
\Phi_{2, \alpha} \\
\Phi_{1, \alpha}
\end{array}\right)=\left(\begin{array}{c}
0 \\
\eta(\neq 0)
\end{array}\right)=b
$$

where $A$ is a matrix with the elements $\left\{A_{i, j}: i, j=1,2\right\}$, and suppose $b=(0, \eta)^{t}$. The existence of the solution above needs $\operatorname{rank}(A)=\operatorname{rank}(A, b)$. After simple but tedious algebraic calculation, we can find that $\operatorname{rank}(A) \neq$ $\operatorname{rank}(A, b)$, when $\eta \neq 0$. So we can conclude that there is no non-zero-energy edge state here. Besides, we can get similar relations with other $\lambda_{i}>1$ and $\lambda_{\bar{i}}<1$. The details are neglected here, all the discussion are rigorous. Thus we rigorously proved that no any edge state exists.

When $m=0$, that leads to $a_{1}=a_{2}=\theta,\left\{\lambda_{i}\right\}$ are still the eigenvalues of transfer matrix $T_{0}$. Because the values of $1 s t$ and $3 r d$ column of $U$ are the same, and those of $2 n d$ and $4 t h$ the same as well, it is easy to get $\operatorname{det} U=0$, so $U^{-1}$ doesn't exist. And it is easy to confirm that $\left|\lambda_{1}\right|=\left|\lambda_{2}\right|=1$ and $\left|\lambda_{3}\right|=\left|\lambda_{4}\right|=1$, which corresponds to the oscillation of the wave function, without decay. Meanwhile, under this condition, we can get $E^{2}=1-\theta^{2}$. Taking this value back to Eq. (44), we can get the relation $2 \cos \left(k_{x} a / 2\right)=$ $\cos \left(\sqrt{3} k_{y} a / 2\right)$. Energies show in Eq. (4) are complete with both real wave vectors, so it results that there are just bulk states when $m=0$.

When $a_{1}^{2}=4$ or $a_{2}^{2}=4, U^{-1}$ does not exist, because the values of 1 st and $2 n d$ column of $U$ are the same. Without loss of generality, we can choose $a_{1}^{2}=4$ in our following discussion, and it is easy to know $m$ is real, so we have $a_{1}=2$. Then we can easily get $\left|\lambda_{1}\right|=\left|\lambda_{2}\right|=1$, while at some $k_{y}$, 
$\left|\lambda_{3}\right|=\left|\lambda_{4}\right|=1$ corresponds to the extended states, and others $\left|\lambda_{3}\right|<1$ and $\left|\lambda_{4}\right|>1$ just $\lambda_{3} \cdot \lambda_{4}=1$. Although $U^{-1}$ doesn't exist, we can suppose to write down it with the definition of right inverse matrix at $a_{1} \rightarrow 2$, so we can find at this limitation, the $1 s t$ and $2 n d$ row of $U^{-1}$ are closely the same in values, while both of them correspond to the extended states. And besides, we can consider the $3 r d$ and $4 t h$ row which corresponds to the possible decay wave with a certain $k_{y}$. Then it is easy to get similar relation as Eq. (18). In order to get the physically meaningful edge states, we can get similar relations as Eq. (19). Considering about a similar inhomogeneous equations, we can conclude that there is no non-zero-energy edge state under this condition. Besides, we can give the similar discussion with $a_{2}=-2$.

In this paper, we give an analytically proof of the non-existence of the edge states in the semi-infinite AEG. By this method, we show the bulk energy spectrum and the condition for the existence of the physically meaningful edge states, according to the rigorous discussion of the transfer matrix and its eigenstates, we can get the necessary and sufficient condition for the existence of zero-energy and non-zero-energy edge states, and finally we find the contradictory condition to show the non-existence of them.

\section{Acknowledgments}

We should like to thank Dr. X.Z. Yan for helpful comments. This work is supported by the National Natural Science Foundation of China under grant No.10847001 and National Basic Research Program of China (973 Program) under the grant (No.2009CB929204, No.2011CB921803) project of China.

\section{References}

[1] R. E. Prange and S. M. Girvin The Quantum Hall Effect (2nd Ed.), (Springer-Verlag, New York, 1990).

[2] R. B. Laughlin, Phys. Rev. B 23, 5632 (1981).

[3] B. I. Halperin, Phys. Rev. B 25, 2185 (1982).

[4] D. P. Arovas, R. N. Bhatt, F. D. M. Haldane, P. B. Littlewood, and R. Rammal, Phys. Rev. Lett. 60, 619 (1988).

[5] C. L. Kane and E. J. Mele, Phys. Rev. Lett. 95, 226801 (2005).

[6] B.A. Bernevig and S. C. Zhang, Phys. Rev. Lett. 96, 106802 (2006).

[7] B. A. Bernevig, T. L. Hughes, and S. C. Zhang, Science 314, 1757 (2006). 
[8] D. J. Thouless, M. Kohmoto, M. P. Nightingale, and M. den Nijs, Phys. Rev. Lett. 49, 405 (1982).

[9] M. Kohmoto, Ann. Phys. (N.Y.) 160, 355 (1985).

[10] Y. Hatsugai, Phys. Rev. Lett. 71, 3697 (1993).

[11] K. S. Novoselov, A. K. Geim, S. V. Morozov, D. Jiang, Y. Zhang, S. V. Dubonos, I. V. Grigorieva, and A. A. Firsov, Science 306, 666 (2004).

[12] Y. Zhang, Y.-W. Tan, H. L. Stormer, and P. Kim, Nature 438, 201 (2005).

[13] A. K. Geim and K. S. Novoselov, Nature Mater. 6, 183 (2007).

[14] D. J. Klein, Chem. Phys. Lett 217, 261 (1994).

[15] K. Nakada, M. Fujita, G. Dresselhaus, and M. S. Dresselhaus, Phys. Rev. B 54, 17954 (1996).

[16] Y. Niimi, T. Matsui, H. Kambara, K. Tagami, M. Tsukada, and H. Fukuyama, Appl. Surf. Sci. 241, 43 (2005).

[17] Y. Kobayashi, K. I. Fukui, T. Enoki, K. Kusakabe, and Y. Kaburagi, Phys. Rev. B 71, 193406 (2005).

[18] S. Ryu and Y. Hastugai, Phys. Rev. Lett 89, 077002 (2002).

[19] K. Wakabayashi, Y. Takane, M. Yamamoto, and M. Sigrist, CARBON 47, 124 (2009).

[20] C. Hu, Phys. Rev. Lett 72, 1526 (1994).

[21] D. H. Lee and J.D. Joannopoulos, Phys. Rev. B 23, 4988 (1981), Phys. Rev. B 24, 6899 (1981).

[22] Liwei Jiang, Yisong Zheng, Cuishan Yi, Haidong Li, and Tianquan Lu, Phys. Rev. B 80, 155454 (2009).

[23] Haidong Li, Lin Wang, Zhihuan Lan, and Yisong Zheng arXiv:0811.3336v1 (2008).

[24] Wei Li, and Ruibao Tao, arXiv:1001.4168v2 (2010).

[25] Yuanyuan Zhao, Wei Li, and Ruibao Tao, arXiv:1101.3411v2 (2011). 